\title{
Médiévales
}

Langues, Textes, Histoire

\section{Le notariat, pratique juridique et sociale : les lieux de souscription des actes à Cavaillon au début du $\mathrm{XV}^{\mathrm{e}}$ siècle}

Social and legal Aspects of Notarial Pratice: Acting Places in the Cavaillon charters of the early fifteenth century

\section{Maëlle Ramage}

\section{(2) OpenEdition}

\section{Journals}

Édition électronique

URL : https://journals.openedition.org/medievales/6116

DOI : 10.4000/medievales. 6116

ISSN : $1777-5892$

Éditeur

Presses universitaires de Vincennes

Édition imprimée

Date de publication : 31 décembre 2010

Pagination : 127-143

ISBN : 978-2-84292-267-2

ISSN : 0751-2708

\section{Référence électronique}

Maëlle Ramage, «Le notariat, pratique juridique et sociale : les lieux de souscription des actes à

Cavaillon au début du xve siècle », Médiévales [En ligne], 59 | automne 2010, mis en ligne le 20 mars 2013, consulté le 23 avril 2022. URL : http://journals.openedition.org/medievales/6116 ; DOI : https:// doi.org/10.4000/medievales.6116 
Médiévales 59, automne 2010, p. 127-143

Maëlle RAMAGE

\section{LE NOTARIAT, PRATIQUE JURIDIQUE ET SOCIALE: LES LIEUX DE SOUSCRIPTION DES ACTES À CAVAILLON AU DÉBUT DU XV' SIÈCLE}

Au début du $\mathrm{Xx}^{\mathrm{e}}$ siècle, l'intérêt des historiens se porte vers les actes notariés comme source de l'histoire du droit et des institutions. Roger Aubenas note en 1931 la place importante qui est donnée à l'histoire du notariat d'un point de vue juridique et social, les études concernant les «pays de droit écrit», et notamment la Provence, se multiplient ${ }^{1}$. Au cours des années 1960, les tenants d'une histoire sociale basée sur des dépouillements sériels s'emparent à leur tour des archives notariales. Leurs travaux ont pour point commun de prendre en considération un type d'acte particulier, dont quelques aspects sont isolés et mis en série. Les actes notariés font alors l'objet d'une exploitation intensive mais ne sont pas pensés dans leur globalité ni comme des contrats engageant dans une démarche concrète le notaire et ses clients. Au contraire, l'historiographie du notariat des vingt dernières années est marquée par un intérêt accru pour la figure du notaire et pour ses relations avec sa clientèle, préoccupations auxquelles s'ajoute une attention récente à son métier, ses exigences et ses implications ${ }^{2}$. En témoignent, entre autres, les actes du colloque tenu sur ce sujet à Aix en 2006 et la thèse de Dominique Bidot-Germa publiée en 2008 qui, par l'étude de la documentation notariale produite dans le Béarn jusqu'au début du XvI siècle, retrace la

1. R. Aubenas, Étude sur le notariat provençal au Moyen Âge et sous l'Ancien Régime, Aixen-Provence, 1931, introduction, p. 4.

2. Voir par exemple J.-L. LAFFont, F. OGer et R. Souriac dir., Histoire sociale et actes notariés, problèmes de méthodologie..., Toulouse, 1989; J.-P. Poisson, Notaires et société, travaux d'histoire et de sociologie notariales, 2 tomes, Paris, 1985-1990 ; J.-L. LAFFonT dir., Problèmes et méthodes de l'analyse historique de l'acte notarié (XV'-XIXe siècles), Actes du colloque de Toulouse, Toulouse, 1991 et O. Redon et F. Menant dir., Notaires et crédit dans l'Occident méditerranéen médiéval, Rome, 2004. 
genèse du notariat comme groupe professionnel et explique quelle place les notaires tiennent dans la société ${ }^{3}$.

L'historiographie se caractérise ainsi par une observation plus précise de la personne et de l'activité du notaire pour aller vers la compréhension de la société à laquelle il appartient. En s'inscrivant dans cette évolution, cet article aborde un aspect de la pratique notariale situé au croisement du droit écrit et des usages de la clientèle du notaire, celui des déplacements effectués par ce dernier pour instrumenter les actes. Nous savons en effet que les notaires se rendent fréquemment auprès de leurs clients, soit à leur domicile soit ailleurs dans la ville et nous pouvons dès lors nous demander ce qui motive ces déplacements. Les raisons peuvent être juridiques, instrumenter un acte dans un lieu précis devenant alors l'une des conditions de sa validité. Elles peuvent également être matérielles, ou encore répondre à une logique sociale qui tendrait à attribuer des valeurs particulières aux lieux de vie des habitants.

Je prendrai comme exemple Cavaillon, cité épiscopale du Comtat Venaissin, au début du $\mathrm{Xv}^{\mathrm{e}}$ siècle. Le choix de cette période tient à l'existence d'un livre d'estime réalisé entre 1414 et 1417 par les membres de la communauté de Cavaillon dans le cadre de l'estimation générale des biens des Comtadins entreprise en $1414^{4}$. Inventaire exhaustif des biens immeubles des habitants organisé suivant un parcours à travers l'ensemble du territoire, ce document a permis de cartographier l'espace urbain préalablement à l'analyse de la documentation notariale contemporaine de la production du livre d'estime ${ }^{5}$. Celle-ci consiste en sept registres écrits par trois notaires: trois par Jacques Michaelis qui couvrent les années 1414, 1416 et 1417, trois également par Pierre de Oxnago pour les années 1414, 1415 et 1417-1418 ${ }^{6}$. En revanche, un seul des registres de Jean Ferraguti est conservé pour cette période; il comprend des actes datés des années 1404 à $1415^{7}$. Le corpus ainsi composé de mille deux cent trente-trois actes ne représente qu'une partie de ceux rédigés alors à Cavaillon; nous savons qu'au moins cinq notaires instrumentaient dans

3. L. Fagion, A. Mailloux et L. Verdon dir., Le Notaire entre métier et espace public en Europe VIII'-XVIII siècle, Aix-en-Provence, 2008; D. BIDOt-Germa, Un notariat médiéval. Droit, pouvoir et société en Béarn, Toulouse, 2008.

4. A.D. de Vaucluse, C 88. Sur l'estimation des biens des Comtadins, voir M. Zerner, Le Cadastre, le pouvoir et la terre. Le Comtat Venaissin pontifical au début du Xve siècle, Rome, 1993.

5. Il s'agit de mon mémoire de master, Écritures et pratiques de l'espace à Cavaillon au début du $X^{e}$ siècle soutenu en 2008 à l'université de Versailles-Saint-Quentin.

6. A.D. de Vaucluse, registres 3 E 32 art. 15 et 3 E 33 art. 71 et 72 pour Jacques Michaelis et $i d$. , registres $3 \mathrm{E} 33$ art. 98,99 et 100 .

7. Id., registre 3 E 33 art. 91. 
cette ville qui comptait sept cent cinquante à mille habitants ${ }^{8}$. Il s'agit exclusivement de registres de brèves dans lesquels les notaires consignent au jour le jour tous les actes qu'ils instrumentent, ce qui nous permet de les suivre dans leur activité.

Nous verrons dans un premier temps quels types de contrats les notaires sont appelés à instrumenter et comment ils concilient les exigences de leur propre métier, conférer aux actes une valeur juridique, et les demandes de leur clientèle. Les lieux de souscription des actes seront analysés, dans une deuxième partie, comme l'un des éléments de cette conciliation.

\section{Pourquoi recourir au notaire?}

La lecture des titres des actes permet d'appréhender les différents contrats dont les habitants demandent l'enregistrement à leur notaire car ils se rapportent au verbe du dispositif qui détermine la nature de l'acte. Par exemple, en souscrivant une obligation (obligatio), le débiteur s'oblige vis-à-vis de son créancier; dans les actes d'achat (emptio) le possesseur du bien le vend à quelqu'un qui en retour l'achète aux conditions énoncées.

Pour mille deux cent trente-trois actes enregistrés sur la période, les notaires ont inscrit cinquante-neuf intitulés différents ; parmi eux, vingt-et-un apparaissent une seule fois et trente-sept ne sont employés qu'à cinq reprises ou moins9 . Ces quelques chiffres suffisent à illustrer la grande diversité de situations dans lesquelles les notaires sont appelés à instrumenter et la difficulté d'en proposer une typologie générale.

Les conjonctions de coordination latines seu et $e t$ sont utilisées dans certains titres d'actes. Le 3 mai 1417, Jacques Michaelis instrumente une «vente ou location» effectuée par Hugues Jausserand ${ }^{10}$. L'utilisation conjointe de plusieurs termes, qui n'est pas réservée exclusivement aux actes multiples, c'est-à-

8. À propos des notaires, voir l'inventaire de la série E aux Archives départementales de Vaucluse et, pour une présentation du notariat à Cavaillon à la fin du Moyen Âge, G. LE DANTEC, "Crédit et source notariale à Cavaillon (XIV $-\mathrm{XV}^{\mathrm{e}}$ siècles). Essai de Typologie», dans O. REDON et F. Menant dir., Notaires et crédit..., Rome, 2004, p. 307-335. M. Zerner, «Le cadastre de Carpentras à l'épreuve des autres sources», dans J.-L. Biget, J.-C. Hervé et Y. ThÉBERT dir., Les Cadastres anciens des villes et leur traitement par l'informatique, Actes de la Table ronde organisée par le Centre d'Histoire Urbaine de Saint-Cloud, Paris-Rome, 1989, p. 131-149, fonde son estimation de la population de Cavaillon sur l'étude des livres d'estime du Comtat Venaissin et le dépouillement de registres notariés du début du Xv siècle. Au livre d'estime sont inscrits trois cent trente-cinq propriétaires.

9. Trente-deux actes ne sont pas intitulés, ils ont bien sûr été comptés, tout comme les vingtsept titres que je n'ai pas pu ou su lire.

10. Registre 3 E 33 art. 72, acte 76, fol. 47, 3 mai, Pro Hugone Jausserani allias Tarro cum nobile Ermessenda Bedoti de Cavallionis locatio seu venditionis. 
dire contenant plusieurs conventions, montre que la frontière entre deux types de contrats est parfois floue et que le notaire ressent le besoin de préciser le sens du contrat passé ${ }^{11}$. Ce type d'imprécisions résultait peut-être parfois d'un désaccord entre les clients et leur notaire à propos de la nature de l'acte passé, le notaire ayant préféré, sous l'injonction des parties, ne pas trancher et inscrire les mots utilisés par chacune d'elles. Cela supposerait une réelle intervention de ces dernières dans la rédaction des actes et une connaissance des termes juridiques, ce qui est envisageable pour une partie de la clientèle. La multitude de titres employés et leur manque de précision parfois sont un premier révélateur des difficultés liées au passage d'un droit en théorie très réglé à son application dans une pratique quotidienne.

Un quart des actes inventoriés fait directement référence à un crédit: ce sont les stipulationes et obligationes. Une fois la dette remboursée, les contractants faisaient de nouveau appel au notaire qui cancellait l'acte ou rédigeait une quittance de dette (quitatio ou appodixia). Des baux de différents types sont instrumentés par les notaires, par ailleurs chargés d'enregistrer les transactions des biens immeubles et parfois meubles ${ }^{12}$. À la suite de ces transferts de propriété, les détenteurs de droits de seigneurie les faisaient valoir et reconnaître devant le notaire ${ }^{13}$. En outre, quarante-deux transactions consistent en dons ou cessions dont la contrepartie, si elle a existé, n'est pas spécifiée. Les Cavaillonnais faisaient aussi mettre par écrit associations et pactes de toutes sortes ${ }^{14}$. Le notaire rédige encore de nombreux actes qui se jouent principalement à l'échelle de la famille, tels que les constitutions de dots, les inventaires et partages de biens et les testaments ${ }^{15}$. Enfin, cent cinq procurationes ont été inscrites dans les registres.

Transactions immobilières, crédits ou actes de famille, les habitants sollicitent le notaire pour lui faire enregistrer chacun de leurs engagements. Car l'engagement, fréquemment complémentaire d'une reconnaissance, constitue le dénominateur commun des actes que les Cavaillonnais font rédiger. Par exemple, les transactions, qui font souvent l'objet d'un paiement différé, engagent

11. Sur ce point voir J. Hilaire, La Science des notaires. Une longue histoire, Paris, 2000, p. 38 .

12. Dans les sept registres nous retrouvons quatre formes de bail: des baux en accapte (51), des arrentements (35), les baux à fâcherie (26), des contrats qui concernent des terres ou des terres plus une maison et des locations (13) qui portent presque exclusivement sur des maisons. On relève plus de cent vingt achats, et huit échanges de propriété, parmi les échanges sont distingués les transactiones, excambii, et permutationes.

13. Les transactions sont très souvent suivies du versement des droits de seigneurie, laudimium, et de la reconnaissance du seigneur, recognitio.

14. Nous trouvons un contrat d'association et vingt-huit pactes.

15. Les actes de famille sont au nombre de soixante-quatorze, y figurent notamment vingtcinq constitutions de dots, neuf reconnaissances du versement de la dot, vingt-cinq testaments et trois partages de biens. 
l'acheteur envers le vendeur et parfois envers le seigneur. Les titres des actes démontrent que les habitants ont recours au notaire en toutes sortes d'occasions. Faire rentrer les engagements pris par les habitants dans les cadres d'une écriture notariale, régie par des formulaires, ne va pas sans poser un certain nombre de difficultés et d'hésitations ${ }^{16}$. Ainsi, l'écriture notariale est un véritable exercice d'adaptation du droit et de la rigueur des formules au quotidien et vice-versa.

\section{L'écriture des actes}

Les rédactions notariées impliquent une description abrégée de l'action sur laquelle elles portent, limitée à la qualification de l'engagement des parties et à son objet. La garantie de leur validité juridique implique quant à elle l'utilisation des formules adaptées et un certain niveau de standardisation de l'écriture, indispensable à leur reconnaissance par d'autres personnes ou institutions.

Le discours de l'acte suit un schéma précis qui sert sa validation. Il se présente comme une narration normalisée. Après l'indication de la date et de la notification, le notaire en vient rapidement à l'exposé du dispositif qui définit le fait juridique et dont l'élément essentiel est un verbe d'action. Ce verbe, qui explique la raison d'être de l'acte (vendre, donner, etc.), engage le commanditaire. Sont également développées les conditions du contrat qui peuvent être plus ou moins longues. Certains baux entraînent par exemple la rédaction de conditions très précises quant à l'entretien de la propriété prise en tenure. Après le dispositif, lui-même empli de formules juridiques, le notaire inscrit les éléments de corroboration de l'acte: il affirme l'avoir instrumenté en tant que personne publique, note le lieu d'enregistrement et cite les témoins présents. Les moyens de validation des actes sont multiples - relation de gestes, de paroles, usage de formules de droit savant, liste de témoins -, ce qui permet au notaire de les utiliser toutes, ce qui est souvent le cas, mais aussi d'adapter son texte à la situation. En effet, derrière une structure régulière, les variations sont multiples et certains actes procèdent d'une rédaction beaucoup moins systématique.

Les actes les plus fréquents sont aussi les plus standardisés. Il y a ainsi peu à dire des reconnaissances ou quittances de dettes; seuls les noms, le montant et l'échéance varient d'un acte à l'autre; les raisons du prêt sont parfois signalées. Les textes de procuration sont aussi très systématiques: le commanditaire y désigne comme représentant de son autorité une ou plusieurs personnes. Pour ce qui est des actes plus complexes, comme les partages de biens, constitutions de dots, actes de vente ou de location, ou encore testaments, le début du protocole est tou-

16. Sur ce point voir J. HilaiRe, Le Régime des biens entre époux dans la région de Montpellier du début du XIII siècle à la fin du XVI siècle, Paris, 1957 et ID., La science des notaires..., op. cit., Paris, 2000. 
jours le même et seul son développement diffère. Dans tous ces types de contrats, la structure connaît peu de variations; en revanche, elles sont beaucoup plus fréquentes dans les actes peu représentés dans le corpus. Cela pourrait s'expliquer par le recours à un formulaire pour les actes les plus courants alors que les contrats plus rares laissent le champ libre à une écriture moins contrainte. La rédaction effectuée par le notaire consiste alors en une narration précise des gestes et paroles des différents protagonistes au cours de laquelle viennent s'intercaler des formules juridiques que le notaire adapte, les rejetant parfois à la fin du texte ${ }^{17}$.

L'attention portée par les notaires aux circonstances de l'énonciation associée aux exigences juridiques explique l'indication précise et presque systématique du lieu de souscription des contrats ${ }^{18}$. Les mentions topographiques peuvent prendre plusieurs formes: ce sont soit des toponymes, soit des points de repères, propriétés privées ou monuments remarquables tels les portes de la ville, les remparts ou encore une église. Le notaire les inscrit en fin d'acte dans une phrase courte de type «Acte passé à Cavaillon sur la Grande Place, sous les arcades» (Actum fuit hoc Cavallionis in Platea subtus arcos) ou bien «Acte passé à Cavaillon dans la maison des héritiers de Jacques Jacobi» (Actum fuit hoc Cavallionis in hospicio heredium dicti quondam Jacobi Jacobi) ${ }^{19}$.

Si la question est relativement absente de la bibliographie, la documentation permet de faire l'histoire des déplacements des notaires ${ }^{20}$. Or celle-ci donne accès à leur usage de l'espace urbain par les habitants. La rédaction même des actes souligne l'importance attachée par le notaire au contexte de discussion des contrats dans une société où se conjuguent droit écrit et gestes coutumiers dans l'expression des rapports sociaux.

17. Voir par exemple registre 3 E 33 art. 71, acte 189, fol. 107v., Pro Universitate Cavallicensi cum Petro de Oxnago notario dicti loci. Le 9 novembre 1416, Jacques Michaelis rédige la nomination de Pierre de Oxnago comme scribe et notaire de la communauté. Il commence par préciser que le conseil se réunit dans la maison du pape et en présence du noble citoyen Charles de Aussaco, viguier. Figure ensuite la liste des syndics et Jacques Michaelis raconte comment et à quelles conditions Pierre de Oxnago est élu.

18. Sur l'ensemble du corpus, 32 lieux de souscription ne sont pas indiqués.

19. Registre 3 E 33 art. 72, acte 138, fol. 94v., 29 septembre 1417 et acte 5, fol. 3 v., 10 janvier 1417.

20. Quelques recherches éditées dans L. Fagion, A. Mailloux, L. Verdon, Le Notaire entre métier et espace public..., Aix-en-Provence, 2008, font appel aux lieux de souscription des actes. Anne Mailloux, «L'émergence du notariat à Lucques (VIII $-\mathrm{X}^{\mathrm{e}}$ siècle), normes et pratiques d'un corps professionnel», p. 19, les analyse pour repérer les lieux de concentration de l'activité notariale. Chantal Amman-Doubliez, «Assit principio sancta Maria meo. Le notaire Jean de Freneto de Sainte Marie d'Agaune (1472), sa figure et son métier», p. 126-128, évalue à partir des lieux de souscription le temps passé par le notaire hors de chez lui. Donatella BARTOLINI propose une analyse des lieux d'enregistrement des actes qui, conjuguée à l'observation des registres du notaire Bassani de Feltre, lui permet de caractériser sa pratique notariale ( «Pratique notariale dans une communauté de la Terre Ferme vénitienne entre les XIV et XVIII ${ }^{\mathrm{e}}$ siècles.», p. 261-262). 


\section{Les lieux de souscription des actes}

Les notaires pouvaient être amenés à se déplacer afin de prendre note des contrats sur un brouillard ou bien directement dans leur registre de brèves. L'état matériel des documents dont nous disposons indique en effet que les notaires, qui travaillaient sur des cahiers de dix à vingt folios qu'ils reliaient ensuite dans des volumes annuels, les emportaient souvent avec eux. Les premières et dernières pages de ces cahiers sont d'ordinaire cornées et sales. Par ailleurs, la graphie très cursive et peu soignée de certains actes peut témoigner de leur écriture hors de l'office. C'est souvent le cas des procurations, contrats fréquents, que le notaire connaissait sans doute par cœur et dont la rédaction n'exigeait pas forcément le recours à un formulaire.

\begin{tabular}{|l|c|c|c|c|}
\hline & $\begin{array}{c}\text { Jacques } \\
\text { Michaelis }\end{array}$ & $\begin{array}{c}\text { Jean } \\
\text { Ferraguti }\end{array}$ & $\begin{array}{c}\text { Pierre de } \\
\text { Oxnago }\end{array}$ & Total \\
\hline Etude notariale & $\mathbf{2 7 3}$ & $\mathbf{6 0}$ & $\mathbf{1 5 2}$ & $\mathbf{4 8 5}$ \\
\hline Domicile & $\mathbf{1 3 8}$ & $\mathbf{2 5}$ & $\mathbf{2 0 4}$ & $\mathbf{3 6 7}$ \\
\hline De l'une des parties & 83 & 13 & 99 & 195 \\
\hline D'un tiers concerné & 17 & 7 & 58 & 82 \\
\hline D'un témoin & 10 & 2 & 19 & 31 \\
\hline D'un tiers non cité & 21 & 3 & 28 & 52 \\
\hline Non lus & 7 & 0 & 0 & 7 \\
\hline À l'extérieur & $\mathbf{9 9}$ & $\mathbf{2 0}$ & $\mathbf{5 2}$ & $\mathbf{1 7 1}$ \\
\hline In Platea & 46 & 10 & 17 & 73 \\
\hline In carreria... & 40 & 2 & 10 & 52 \\
\hline Ante... & 13 & 8 & 25 & 46 \\
\hline Lieu ecclésiastique & 64 & 8 & 43 & 115 \\
\hline Palais épiscopal & 42 & 5 & 20 & 67 \\
\hline Réfectoire des chanoines & 4 & 1 & 9 & 14 \\
\hline Cathédrale & 17 & 0 & 4 & 21 \\
\hline Monastère St-Jean & 1 & 2 & 5 & 8 \\
\hline Église St-Étienne & 0 & 0 & 5 & 5 \\
\hline Hors de Cavaillon & $\mathbf{1 2}$ & $\mathbf{0}$ & $\mathbf{9}$ & $\mathbf{2 1}$ \\
\hline Non lus ou manquant & $\mathbf{6}$ & $\mathbf{9}$ & $\mathbf{2 7}$ & $\mathbf{4 2}$ \\
\hline Non renseignés & $\mathbf{4}$ & $\mathbf{9}$ & $\mathbf{1 9}$ & $\mathbf{3 2}$ \\
\hline Total & $\mathbf{5 9 4}$ & $\mathbf{1 3 1}$ & $\mathbf{5 0 6}$ & $\mathbf{1 2 3 3}$ \\
\hline & & & & \\
\hline
\end{tabular}

Lieux d'enregistrement des actes notariés. 
Restitution de la ville de Cavaillon au $\mathrm{Xv}^{\mathrm{e}}$ siècle

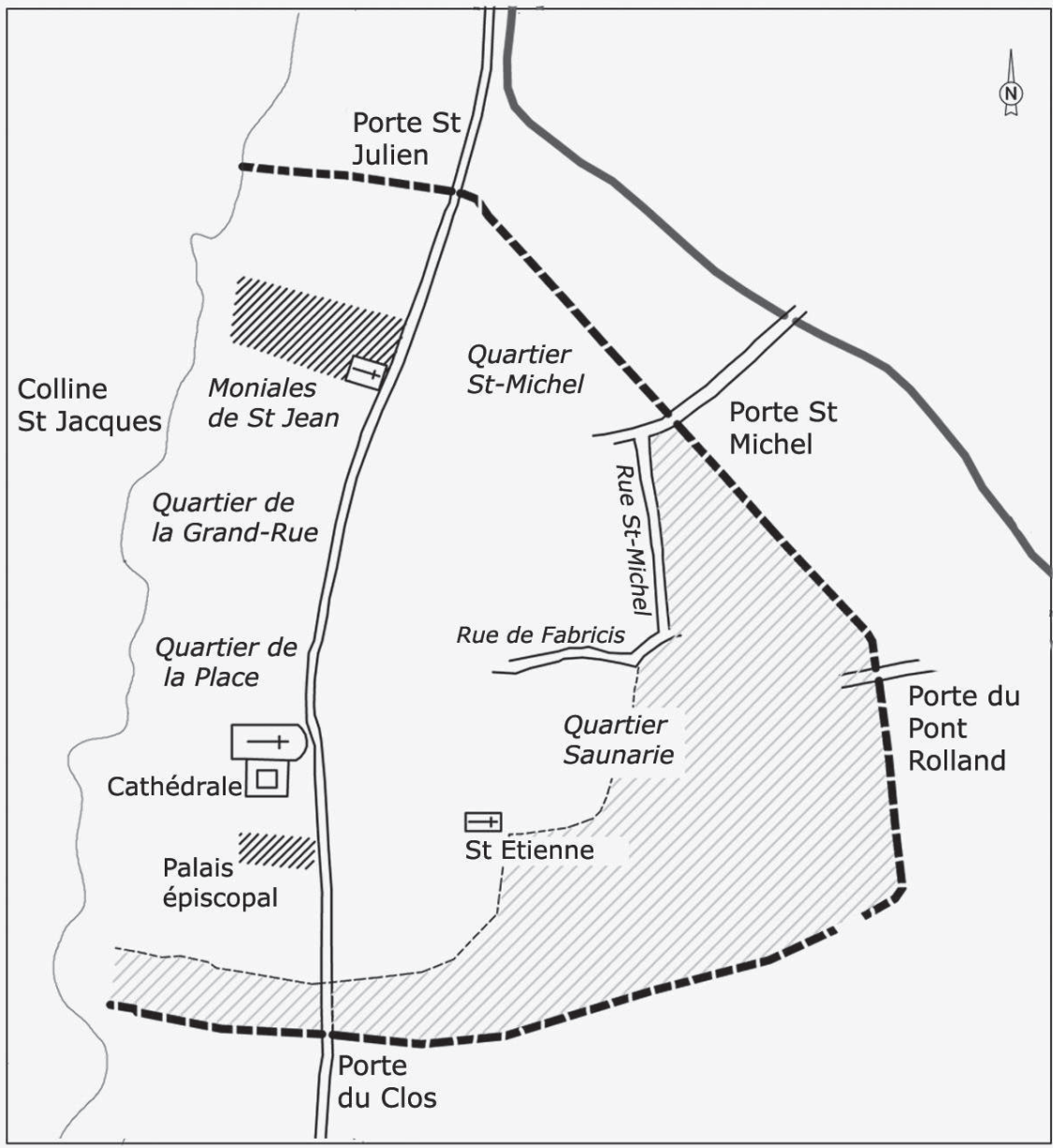

Anciens faubourgs (XIIIe - XIVe siècles)

Emprise présumée des propriétés

Tracé du mur d'enceinte du XIVe siècle 
Les notaires instrumentent $40 \%$ des actes dans leurs études qui se trouvent toutes dans le quartier de la Platée ${ }^{21}$. Les habitants viennent notamment y contracter plus de la moitié des reconnaissances de seigneurie (laudimia), mais cela ne signifie pas qu'ils se déplacent expressément dans ce but. En effet, ces reconnaissances sont pour la plupart consécutives à des achats ou des baux enregistrés dans l'étude ${ }^{22}$. Près de la moitié des procurations sont contractées chez les notaires, des pactes de toutes sortes, dix-neuf sur les vingt-sept et, en bien moindre proportion, des quittances, dons ou échanges de biens et un testament. Nous retrouvons encore plus de la moitié des crédits et des achats, actes qui ont en commun le transfert d'une somme d'argent d'un contractant à l'autre ${ }^{23}$. Se rendre chez le notaire pourrait alors être lié à la nécessité de compter les devises. Pour autant, l'étude n'est pas un lieu où l'on se rend systématiquement pour régler les questions économiques et financières.

S'ils sortent rarement de Cavaillon - seuls Jacques Michaelis et Pierre de Oxnago instrumentent un total de vingt-et-un actes dans une autre localité-, les notaires parcourent tous l'ensemble de la ville. Nous avons vu que plus de la moitié des actes qu'ils enregistrent le sont en dehors de leurs études ${ }^{24}$. Ils se rendent notamment très régulièrement au domicile de leurs clients, et certains contrats sont même passés dans la rue ou encore dans des lieux ecclésiastiques. Tous ces déplacements effectués par les notaires délimitent l'étendue géographique couverte par leurs études. En retour, ils nous indiquent la manière dont certaines activités quotidiennes des habitants prennent place dans l'espace urbain, contribuant ainsi à le qualifier.

21. A.D. de Vaucluse, C88, L'étude de Pierre de Oxnago donne sur la Grande Place, tandis que celle de Jacques Michaelis est située non loin de là, derrière la cathédrale, la situation de l'étude de Jean Ferraguti dans ce même quartier n'est pas précisée.

22. Registres 3 E 32 art. 15 et 3 E 33 art. 71, 72, 91, 98, 99 et 100. Quarante-trois reconnaissances de seigneurie, sur les quatre-vingt-quatorze que compte le corpus, sont enregistrées chez le notaire, parmi elles trente-cinq succèdent à un bail ou un achat enregistré dans le même lieu. Par exemple lorsque, le 12 mars 1414, Véran de Aligno vend une vigne à Elzéar Gasqui, Jean Ferraguti enregistre ensuite la reconnaissance par ce dernier de la seigneurie d'Antoine Ruffi. Registre 3 E 32 art. 15, actes 34 à 36, fol. 196v. 197.

23. Id., Il est à noter ici que pour quarante actes de crédit le lieu de souscription est manquant ou illisible.

24. Registres 3 E 32 art. 15 et 3 E 33 art. 71 et 72 . Jacques Michaelis se rend huit fois à Ménerbes et Orgon. Pierre de Oxnago enregistre neuf actes à Maubec, les Taillades, l'Isle et Ménerbes, ses déplacements de 1417 sont liés à sa fonction de notaire de la communauté. Les deux hommes se déplacent dans les localités voisines exclusivement pour enregistrer des actes concernant des Cavaillonnais qui font partie de leur clientèle. Certains habitants semblent donc préférer se déplacer avec leur notaire au lieu d'en trouver un sur le lieu de leur destination. 
Les notaires se rendent au domicile de leurs clients pour enregistrer $30 \%$ des actes et utilisent alors trente-neuf titres différents ${ }^{25}$. La maison est incontestablement le lieu du dialogue autour des engagements ayant trait à la famille : toutes les constitutions de dots y sont contractées, majoritairement chez les parents du futur marié, sanctionnant l'alliance de deux maisons. $30 \%$ des actes de donations qui mettent en jeu des rapports de solidarité difficilement définissables sont instrumentés chez les habitants ${ }^{26}$. En outre le notaire effectue vingtdeux des vingt-cinq testaments, les trois partages et les six inventaires de biens de particuliers chez ses clients. Cela s'explique par la situation dans laquelle ces actes sont contractés - urgence, bien souvent, dans le cas du testament et nécessité de voir les biens visés par les inventaires et partages de biens -, mais la venue du notaire est aussi révélatrice de son implication dans les contrats audelà de la simple mise en forme de l'acte.

Le domicile est en outre investi pour des discussions et des prises de décisions relatives à l'économie. $20 \%$ des actes de crédit, de transferts de propriété et des baux de toutes sortes y sont instrumentés. Notons que pour le tiers des ventes de propriétés situées dans la ville, acheteurs, vendeurs et notaires se retrouvent dans la maison objet de la transaction. Sans doute l'enregistrement de l'acte s'accompagne-t-il alors de la remise effective de la propriété à l'acheteur ${ }^{27}$. À la suite des transactions, dix-huit des trente-deux revendications et reconnaissances de seigneuries sont instrumentées non chez l'un des acteurs de la transaction, mais chez le détenteur des droits. La variété des situations traitées dans la maison renvoie à ses multiples attributions: lieu de la vie familiale et des solidarités, elle est aussi celui des activités économiques et de l'expression des rapports d'obligation.

Cent quatorze actes sont enregistrés dans des lieux ecclésiastiques: palais de l'évêque, cathédrale Saint-Véran, réfectoire des chanoines, monastère des moniales de Saint-Jean ou encore chapelle Saint-Étienne. Ces contrats portent le plus souvent sur la gestion des biens des institutions ecclésiastiques. $56 \%$ d'entre eux sont discutés dans le palais épiscopal ; il s'agit pour l'essentiel de reconnaissances de la seigneurie de l'évêque et de versement des droits associés par des tenanciers venus à sa rencontre. Jacques Michaelis et Pierre de Oxnago se rendent dans la cathédrale, son cloître ou le réfectoire du chapitre qui est donc accessible aux laïcs ${ }^{28}$. Lorsque les moniales de Saint-Jean pas-

25. Registres 3 E 32 art. 15 et 3 E 33 art. 71, 72, 91, 98, 99 et 100.

26. Ces actes sont intitulés donatio, cessio, datio insolitum, receptio,...

27. Registres 3 E 32 art. 15 et 3 E 33 art. 72, 91, 98, 99 et 100. Le lieu de souscription est alors indiqué comme suit: Actum fuit hoc Cavallionis in hospicio supra vendito ou supra dicto.

28. Registre 3 E 32 art. 15, acte 140, fol. 65 et registre 3 E 33 art. 99, actes 143 à 147, fol. 71-74v, le 25 novembre 1415, Pierre de Oxnago instrumente cinq actes dans le réfectoire: deux donations, deux procurations et le bail d'une terre. 
sent un contrat avec des habitants, exclusivement des baux et des transactions, le notaire et ces derniers se déplacent systématiquement au monastère ou dans son église. Les actes contractés dans les institutions ecclésiastiques suivent la même logique que ceux contractés chez les laïcs, ils sont en revanche d'une moins grande variété et plus axés sur la mise par écrit de la gestion d'une propriété souvent considérable.

Les notaires ont instrumenté plus de cent soixante actes à l'extérieur, que ce soit sur la Grande Place, située à proximité de la cathédrale, dans une rue ou une traverse. Parmi ces actes figurent des transactions, échanges, cessions, reconnaissances de seigneuries, quelques crédits et des négociations. Cela témoigne à première vue d'un réel investissement de l'espace urbain par les habitants, mais celui-ci ne doit pas pour autant être considéré comme un espace public indifférencié.

Vingt et un des actes instrumentés à l'extérieur le sont par Jacques Michaelis devant son étude et deux par Pierre de Oxnago devant la sienne ${ }^{29}$. Nous pouvons alors penser que les clients sont allés trouver le notaire comme lorsque les contrats sont passés à l'intérieur de l'étude. De même, bien souvent les notaires enregistrent des actes devant la maison de l'une des parties ou d'un témoin. Par exemple, le 4 mars 1417, Jacques Michaelis prend acte d'un achat de laine devant la maison de l'acheteur ${ }^{30}$. La fréquence des contrats conclus devant les habitations et la relation entre le lieu de souscription et les contractants suggèrent que cet espace est considéré comme une continuité de la propriété privée. Mais s'il existe une typologie particulière des actes enregistrés à l'intérieur de la maison, cela n'est plus vrai pour ceux qui le sont devant la maison; ils relèvent des mêmes catégories que l'ensemble des actes contractés à l'extérieur.

L'observation plus détaillée des contrats discutés dans la rue fait apparâ̂tre une réelle volonté de publicité de la part de certains contractants. L'évêque, qui d'ordinaire ne contracte aucun acte hors de son palais, fait enregistrer le 12 avril 1415 une reconnaissance de dette de quelques sous devant un four qui se trouve à proximité de sa demeure ${ }^{31}$. Nous pouvons dès lors penser que l'objectif du prélat est moins de réclamer une somme dérisoire que d'affirmer sa position en dévoilant l'existence d'une dette dans un lieu qui devait être fréquenté. L'endroit choisi par le juge pour entendre un habitant le 14 février 1415 n'est certainement pas lui non plus le fait du hasard. Pierre de Oxnago précise que la séance se tient sur la voie publique devant la porte de notre seigneur le pape (in carreria publica ante portam domini nostri pape). Le juge du Comtat

29. Registres 3 E 32 art. 15 et 3 E 33 art. 71, 72, et 100.

30. Registre 3 E 33 art. 72, acte 27, fol. 16.

31. Registre 3 E 33 art 100, acte 50, fol. 20. 
Venaissin tient son audience devant une maison appartenant à la papauté qu'il représente ${ }^{32}$. C'est sur la Grande Place qu'un notable, Elzéar Romey, consent l'unique réduction de cens inscrite dans les registres à l'un de ses tenanciers, le 24 mars $1415^{33}$. C'est de nouveau sur cette place que Raymond Cabassole, syndic, et Raymond Porquerie, percepteur des redevances dues à l'évêque, discutent de leur désaccord avec les représentants de la communauté de l'Isle à propos des redevances ${ }^{34}$. Or les contrats instrumentés à l'extérieur désignent la Grande Place, dans le quartier du même nom, comme un lieu central de la vie sociale. Les soixante-treize actes qui y sont enregistrés par les trois notaires laissent en effet deviner un lieu très fréquenté. De plus, le marché se tient sous les arcades qui bordent la place.

Il serait difficile de donner en quelques mots une synthèse qui ne soit pas trop simplificatrice des déplacements des notaires auprès de leurs clients en regard des types d'actes que ces derniers leur demandent d'instrumenter. En effet, seuls les actes de famille sont systématiquement enregistrés dans la maison de l'une des parties; celle des parents du futur époux lors d'une constitution de dot, celle du donateur lors d'une cession. Mais si l'on sort de ce cadre, le lien entre le type d'acte et le lieu de son enregistrement n'apparaît pas directement. Nous avons vu que la diversité des contrats dont le notaire prend note chez ses clients était presque égale à celle du corpus dans son ensemble, que les habitants se rendent chez leur notaire pour des contrats aux implications économiques mais aussi pour des reconnaissances ou des procurations, ou encore que les notaires emploient vingtsix titres différents afin de nommer les actes enregistrés à l'extérieur.

Si une règle du jeu préside au choix d'un lieu pour telle ou telle discussion, elle ne réside donc pas dans l'attribution d'une fonction particulière à chaque espace. En outre, aucune frontière définitive ne semble venir séparer les sphères du privé et du public. Une continuité réelle existe entre la maison ou l'étude notariale et la rue ou la place. En revanche, les usages des habitants montrent qu'ils n'accordent pas à tous les lieux la même valeur. Ainsi, la Grande Place est perçue comme apte à recevoir les discussions concernant l'ensemble de l'universitas des habitants ou celles auxquelles on souhaite donner une certaine publicité garantie par la présence de nombreuses personnes, qui ne sont pas directement impliquées lors de l'instrumentation du contrat. Ce seraient alors la présence de nombreuses personnes et l'usage fait du lieu par les dirigeants de la ville - syndics pour y traiter des affaires de la communauté, seigneurs pour y régler des questions de droits - qui conféreraient à la Grande Place son caractère public. Son statut juridique ne suffit pas à la caractériser.

32. Registre 3 E 33 art. 99, acte 22, fol. 9.

33. Registre 3 E 33 art. 91, acte 30, fol. 233 .

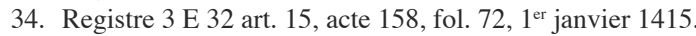


En retour, les espaces choisis par les habitants pour telle ou telle action contribuent à les qualifier.

\section{Choix des lieux de souscription : essai d'interprétation}

Selon Donatella Bartolini, dont l'analyse des lieux de souscription des actes enregistrés par un notaire de Feltre, Bassani, aboutit au même constat de leur multiplicité, nous avons là la marque «d'un style [dans l'exercice du notariat par Bassani] qui est celui de l'occasionnel et du manque de solennité dans la rédaction des actes ${ }^{35}$. $\gg$ La diversité des lieux de souscription serait en partie liée au peu d'importance attachée à la mise par écrit des contrats par un notaire, par ailleurs occupé à d'autres activités. En ce qui concerne les notaires de Cavaillon, nous savons que le rythme d'enregistrement des actes est relativement faible et que chacun d'entre eux complète ses revenus par une ou plusieurs activités ${ }^{36}$. Ainsi, l'explication formulée par Donatella Bartolini pourrait aussi bien s'appliquer à Cavaillon mais elle n'accorde pas, me semble-t-il, suffisamment de place à l'influence de la clientèle sur une pratique notariale qui est également une réponse à une demande formulée par les habitants d'enregistrement d'accords dont ils veulent garder la trace. Le métier du notaire et, par conséquent, le choix des lieux d'instrumentation sont pris dans une logique sociale; en aucun cas leur multiplicité ne relève d'une indifférenciation, voire du seul bon vouloir du notaire et du degré de son implication. Le processus d'écriture des actes ne peut être pensé en dehors de l'organisation de la société qui les demande. Sur ce point, l'étude conjuguée des déictiques avec celle des titres des actes - dont nous avons vu qu'ils renvoient directement à la portée du contrat - et de la position sociale des contractants indique que le choix du lieu réside dans une adéquation entre ces différents paramètres.

Les reconnaissances de seigneuries illustrent clairement l'importance du

35. D. Bartolini, «Pratique notariale dans une communauté de la Terre Ferme vénitienne entre les XIV et XVII ${ }^{e}$ siècles » dans L. Fagion, A. MaIlloux et L. Verdon, Le Notaire entre métier et espace public..., p. 261-262. Ici, l'étude des lieux de souscription est employée à la définition d'une manière d'exercer par les notaires de Feltre. L'auteur appuie également son constat sur l'usage par les notaires de registres au format «de poche» composés de matériaux pauvres et sur le protocole réduit qui fait l'objet de la rédaction.

36. Registres 3 E 32 art. 15 et 3 E 33 art. 71, 72, 91, 98, 99 et 100. Entre 1414, 1416 et 1417 Jacques Michaelis enregistre un peu plus de six cents actes, il ne reste de Jean Ferraguti que cent trente et un actes enregistrés entre fin janvier 1414 et mars 1415, les registres laissés par Pierre de Oxnago comptent cinq cent dix-neuf actes. Les notaires enregistrent donc moins d'un acte par jour en moyenne. Ils sont par ailleurs prêteurs, gestionnaires ou exploitants de plusieurs parcelles de terre ou encore notaires de la communauté ou de la curie épiscopale. 
geste, entendu au sens large, qui concourt à donner forme à l'engagement entre les parties. Lorsque ces reconnaissances ne sont pas instrumentées dans l'office du notaire, elles le sont au domicile du seigneur s'il s'agit d'un laïc. S'il est clerc, la reconnaissance peut prendre place soit à son domicile, soit dans un lieu se rapportant à sa fonction ecclésiastique. Grâce à l'enregistrement chronologique des actes dans les registres de brèves, nous pouvons comprendre leurs successions et les parcours dans la ville suscités par les contrats. Ainsi, les reconnaissances sont toujours précédées d'une transaction ou, de préférence, d'un bail conclu chez l'une des parties. Par exemple, le 12 février 1415, Pierre de Oxnago instrumente l'achat d'une maison appartenant à Jean Maurani et à sa femme par Jacques Raymundi dans ladite maison ${ }^{37}$. Ensuite, le petit groupe accompagné des témoins de la vente se rend chez Pierre Augeri qui fait alors valoir ses droits seigneuriaux que Jacques Raymundi reconnaît ${ }^{38}$. La cession de la propriété est ici divisée en trois étapes, auxquelles correspondent trois actes: emptio, puis laudimium et recognitio, et deux lieux particuliers. Ce cas de figure, que nous retrouvons dans $60 \%$ des ventes de propriété, montre que le choix du lieu se fait en accord avec la portée du contrat. L'écriture notariale associée aux déplacements dans la ville jusqu' au domicile du seigneur participe à l'affirmation du statut social de ce dernier. La pratique, d'autant plus visible lorsque les seigneurs sont des clercs, justifie que certaines transactions soient signées dans des lieux appartenant à une institution religieuse, sans que celle-ci ne soit directement concernée. Ainsi, le $1^{\mathrm{er}}$ avril 1415, Pierre de Oxnago enregistre une suite d'actes concernant la vente d'une terre par un habitant de Cavaillon à un autre dans l'église Saint-Étienne en présence d'Étienne Pellegrini, chapelain de Saint-Étienne et seigneur de cette parcelle ${ }^{39}$. L'achat conclu, le chapelain fait valoir des droits de seigneurie qui sont reconnus par Mondonus Carrerie, acheteur. Puis acheteur et vendeur restent dans l'église pour contracter une reconnaissance de dette.

Dans le même sens, les nombreux déplacements des habitants de Cavaillon au palais de l'évêque pour le reconnaître comme seigneur ou faire enregistrer des actes exceptionnels manifestent son autorité sur les clercs comme sur les laïcs.

Nous avons vu que de nombreux actes de portée économique sont instrumentés chez des particuliers. Dans ces cas, c'est bien souvent chez le vendeur ou chez le bailleur que l'accord est établi. Par exemple, le 17 février 1415, Antoine Belloni se trouve chez Charles de Aussaco pour reconnaître sa dette envers lui ${ }^{40}$.

37. L'absence de précision concernant la date d'entrée en possession ne permet pas de savoir si les parties se rendent dans la maison vendue afin de sanctionner sa remise immédiate à l'acheteur.

38. Registre 3 E 33 art. 99 , actes 17,18 et 19 , fol. 7 .

39. Id., actes 41 à 45, fol. 16-17v.

40. Registre 3 E 33 art. 99, acte 43, fol. 19v., Obligatio nobilis Caroli de Aussaco habitantis Cavallionis. 
De même, Pierre de Oxnago se rend chez Raymonde la Pauta lorsqu'elle arrente une terre à Bertrand Bleyni en $1417^{41}$. Ici encore, le contractant que l'acte oblige reconnaît la situation autant par la rédaction d'un acte que par son déplacement au domicile de l'autre personne impliquée. Si les exceptions sont relativement nombreuses - elles représentent près du quart des actes de ce type-, elles s'expliquent facilement par des motifs matériels. Par exemple, lorsqu'Augier Chabaudi prête de l'argent à Huguette Bernarde, la reconnaissance de dette est enregistrée chez celle-ci ${ }^{42}$. L'explication tient certainement à la résidence aux Taillades de Augier Chabaudi et au fait qu'il se déplace régulièrement à Cavaillon pour voir son notaire ${ }^{43}$.

Enfin, il est surprenant de constater que $41 \%$ des actes enregistrés chez les particuliers le sont chez un tiers non contractant ${ }^{44}$. En ce qui concerne les actes de famille, dont $30 \%$ sont instrumentés chez des tiers, il est vraisemblable que ceux-ci sont dans une large proportion des proches ou des parents. Par exemple, les notaires enregistrent quatre testaments dans une maison qui n'est pas celle du testateur, mais la personne qui détient la maison est systématiquement citée parmi les héritiers ${ }^{45}$. Le lien entre la personne chez laquelle l'acte est contracté et l'affaire traitée peut aussi tenir à sa fonction. Les reconnaissances de seigneurie du pape, coseigneur de la ville avec l'évêque, sont ainsi instrumentées chez Jacqueline de Aussaco car elle est chargée de collecter le trezain qui doit être versé au pontife au moment d'une transaction par le nouveau propriétaire. La maison est donc aussi le lieu d'exercice de leurs charges par certains notables et ainsi symbole de leur domination sociale et économique. La moitié des actes enregistrés au domicile d'une personne qui n'est ni contractante ni témoin le sont chez Huguette Eudesse, Jean Veysseri, Antoine Ruffi ou Véran Michaelis, dont vingt-cinq chez ce dernier. Ces quatre personnes apparaissent dans les registres notariés comme des notables. Souvent cités comme témoins, ils sont par ailleurs à l'origine de nombreux contrats de location ou de crédits qui les mettent en relation avec plusieurs tenanciers et débiteurs. De plus, ils font tous partie des propriétaires importants inscrits au livre d'estime de 1414, et les trois hommes sont ou ont été syndics de la communauté. Il sem-

41. Registre 3 E 33 art. 100, acte 25, fol. 15, 2 mars.

42. Registre 3 E 33 art. 98 , acte 14 , fol. 6,8 février.

43. Le village des Taillades est limitrophe de Cavaillon.

44. Registres 3 E 32 art. 15 et 3 E 33 art. 71, 72, 91, 98, 99 et 100, soit cent cinquante actes sur trois cent soixante-sept. Il ne faut pas cependant oublier que dans de nombreux cas il est impossible de déterminer l'existence d'un rapport de parenté entre les personnes citées par le notaire, à moins d'une étude prosopographique exhaustive.

45. Par exemple, registre 3 E 33 art. 91, acte 38 fol. 197, le 19 mars 1415, Doucette, veuve de Guillaume Rostagni, dicte son testament à Jean Ferraguti dans la maison de Clément Castellani, l'un des héritiers désignés. 
blerait donc que ces actes soient passés chez des personnes qui, par leur statut social, possèdent la capacité de corroborer le contrat.

La multiplicité des lieux de souscription ne découle donc pas seulement d'un art de faire du notaire mais aussi de choix de la part des clients qui expriment leurs positions sociales. L'évêque, par exemple, ne se déplace jamais chez l'un de ses tenanciers ou chez qui que ce soit d'autre; au contraire, il reçoit chez lui les habitants, signifiant ainsi les rapports de clientèle conséquents à la gestion de sa seigneurie et à l'exercice de son autorité. Cette coïncidence entre le statut social des parties, le type de lien que l'acte institue ou renforce entre eux, et le lieu d'enregistrement montre que les gestes et les paroles s'associent à l'écriture juridique du texte dans le scellement du contrat et la reconnaissance de ses implications. Cela ne peut qu'influencer l'exercice du notariat et se retrouve dans l'écriture des documents.

Le notaire ne se contente pas de donner une force probatoire aux actes en y inscrivant les formules de droit adaptées. Pour chaque contrat passé, il précise au contraire les circonstances, les paroles prononcées et le lieu choisi pour cet échange. Il existe donc bien une histoire des déplacements des notaires qui permet de faire une étude concrète du notariat, de ses rapports avec la clientèle et avec l'espace urbain. Ici, les déplacements des notaires à la rencontre de leurs clients relèvent d'une logique sociale reposant sur le statut des contractants, sur la portée des actes et la valeur accordée aux lieux.

Partir d'un détail, les lieux de souscription, a également pour objectif de redonner au notariat sa dimension anthropologique, souvent oubliée par l'histoire institutionnelle. La pratique notariale est en effet juridique mais elle est aussi sociale, ces deux aspects étant intimement liés: l'application du droit est ellemême prise dans une série d'habitudes qui l'inscrivent dans un milieu social.

Maëlle Ramage, LAMOP, Université Paris 1 Panthéon-Sorbonne, 5 rue Victor Cousin, 75005 Paris. 


\section{Le notariat, pratique juridique et sociale : les lieux de souscription des actes à Cavaillon au début $\mathbf{d u} \mathbf{x v}^{\mathrm{e}}$ siècle}

L'historiographie du notariat des vingt dernières années se caractérise par l'observation plus précise de la personne et de l'activité du notaire pour aller vers la compréhension de la société à laquelle il appartient. S'inscrivant dans cette évolution, cet article propose une analyse des déplacements effectués par quelques notaires de Cavaillon au début du $\mathrm{XV}^{\mathrm{e}}$ siècle dans le but d'instrumenter les actes. Le corpus de l'étude consiste en sept registres écrits par trois notaires entre 1414 et 1417. Il s'agit exclusivement de registres de brèves dans lesquels les notaires consignent au jour le jour tous les actes qu'ils instrumentent, ce qui nous permet de les suivre dans leur activité. Le notaire ne se contente pas, en effet, de donner une force probatoire aux actes en y inscrivant les formules de droit adaptées, mais précise les circonstances, les paroles prononcées et le lieu choisi pour cet échange. L'étude de ces éléments montre que la diversité des lieux de souscription ne découle pas seulement des règles de l'exercice notarial mais aussi de choix de la part des clients. Ici, les déplacements des notaires à la rencontre de leurs clients relèvent d'une logique sociale reposant sur le statut des contractants, sur la portée des actes et la valeur accordée aux lieux.

Notariat - ville - espace urbain - Comtat Venaissin

\section{Social and legal Aspects of Notarial Pratice: Acting Places in the Early 15th century Cavaillon charters}

In the past twenty years, medievalists have strived to focus more accurately than before on the personality and activity of notaries in order to get a better understanding of the society to which they belong. Following this logic, I propose to study the movements of three notaries of Cavaillon in the early 15 th century through the pattern of places mentioned as the places where the deeds were acted. Seven registers dating between 1414 and 1417 were studied - registers of day to day recording what was de facto legally acted as well as the circumstances of the agreement, words spoken on the occasion and also the place where it took place. The study reveals that the notary often went to a place chosen by his clients, and that this logic was governed by the social status of the parties, the relative importance of the deed and the symbolics of space.

Notary - town/city - urban space - Comtat Venaissin

Traduction: Harmony Dewez 
